\title{
Electrical Characterizations of Schottky Diodes on ITO Modified by Aromatic SAMs
}

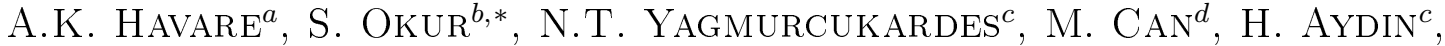 \\ M. SEKER $^{e}$ AND S. DEMIC ${ }^{b}$ \\ ${ }^{a}$ Toros University, Faculty of Engineering, Mersin, Turkey \\ ${ }^{b}$ Izmir Katip Celebi University, Material Science and Engineering, Çiğli, İzmir, Turkey \\ ${ }^{c}$ Izmir Institute of Technology, Faculty of Science, Material Science and Engineering, Izmir, Turkey \\ ${ }^{d}$ Institute of Solar Energy, Ege University, Izmir, Turkey \\ ${ }^{e}$ Ondokuz Mayıs University, Science and Arts Faculty, Department of Physics, Samsun, Turkey
}

\begin{abstract}
In order to understand the electronic properties of the organic Schottky diode, ITO/TPD/Al and ITO/SAM/ TPD/Al organic Schottky devices were fabricated to obtain current-voltage characteristics. From the slopes and $y$-axis intercepts of the plots, the values of the ideality factor, barrier heights of the ITO/SAM/TPD/Al diode were determined as 2.03 and $0.56 \mathrm{eV}$, respectively. The surface characterizations of modified and unmodified ITO were performed via atomic force microscopy.
\end{abstract}

DOI: 10.12693/APhysPolA.123.456

PACS: 73.20.Hb, 73.20.Mf, 73.21.-b, 73.22.-f, 73.25.+i, 73.40.Ei, 73.40.Jn, 73.40.Ns

\section{Introduction}

The organic semiconducting molecules, having conjugated $\pi$-electrons, have had a wide potential for electronic devices applications since the discovery of conducting polymers [1]. The organic semiconductors and their derivatives are used as active components in the Schottky diode in recent years [2]. The rectification performance of the diodes depends on their electronic characteristics and interface properties [3]. The difference between work functions of anode electrode and the adjacent organic layer determines the Schottky barrier properties at the metal/organic interface. Therefore, modification of the surface of the anode material, indium tin oxide (ITO), with self-assembled monolayer (SAM) molecules is one of the most convenient methods to improve electronic parameters of the Schottky diode in terms of barrier height, ideality factor and work function [4].

In literature, diodes of composite organic semiconductors with polyaniline with polystyrene and ITO $/ \mathrm{C} 70 / \mathrm{Au}$ have been investigated using the Schottky diode technique [5]. Siloxane-derivatized hole injector (TPD-Si ${ }_{2}$ ) was synthesized and used as SAM to improve the charge injection. But the results show that triethoxysilyl with alkyl chain as spacer of the charge injection from ITO surface is decreased because of non-conducting alkyl spacers [6].

In our study, SAM is used to modify the ITO surface in order to constitute a better alignment between the work functions of anode and the organic layers at the interface. $\quad 4$-[(3-methylhphenyl)(phenyl)amino]benzoic acid (MPPBA) and (4'-iodobiphenyl-4-yl) trihydroxysilane

*corresponding author
(THIBSi) have been used as SAMs materials. In order to compare the effect of aromatic SAM molecules on the electronic parameters of the Schottky diodes, we fabricated ITO/TPD/Al, ITO/MPPBA/TPD/Al and ITO/ THIBSi/TPD/Al devices. Electrical parameters of the devices were investigated using forward bias currentvoltage measurements.

In the Schottky injection mechanism, when adequate thermal energy needed to cross the barrier height is acquired, electrons from the metal electrode can be injected. The process can be described by the equation [7],

$$
J=\left(\frac{4 \pi q m k}{h^{3}}\right) T^{2} \exp \left(\frac{-q \phi_{\mathrm{B}}}{k T}\right)\left[\exp \left(\frac{q V}{k T}-1\right)\right],
$$

where $q$ is the electron charge, $m$ is the effective mass of the electron or hole, $k$ is the Boltzmann constant, $h$ is the Planck constant, $T$ is the temperature, $\phi_{\mathrm{B}}$ is the barrier height and $V$ is the applied voltage. The current-voltage characteristics for $q\left(V-I R_{\mathrm{S}}\right)>k T$ values of the diodes can be analyzed by the relation [8],

$$
I=I_{0} \exp \left(\frac{q\left(V-I R_{\mathrm{S}}\right)}{n k T}\right)\left[1-\exp \left(\frac{q\left(V-I R_{\mathrm{S}}\right)}{k T}\right)\right],
$$

where $I_{0}$ is the saturation current and is expressed as

$$
I_{0}=A A^{*} T^{2} \exp \left(-\frac{q \phi_{\mathrm{B}}}{k T}\right),
$$

where $R_{\mathrm{S}}$ is the series resistance, $q$ is the electron charge, $V$ is the applied voltage, $A^{*}$ is the effective Richardson constant, $A$ is the effective contact area, $T$ is the absolute temperature, $k$ is the Boltzmann constant, $n$ is the ideality factor. The saturation current obtained from the linear portion intercepts $\log I$ at zero voltage. The ideality factor and barrier height values of the four diodes were calculated from the slope of the linear region and 
$y$-axis intercept of the forward bias $\ln (I)-V$ curve. The obtained ideality factors higher than unity indicate that diodes show non-ideal behavior because of the interface layer and series resistance. This significant series resistance effect can be analyzed by Cheung's functions [9]:

$$
\frac{\mathrm{d} V}{\mathrm{~d} \ln I}=n \frac{k T}{q}+I R_{\mathrm{S}}
$$

and $H(I)$ function can be defined as follows:

$$
H(I)=V-n \frac{k T}{q} \ln \left(I_{0} \frac{A A^{*}}{T^{2}}\right)=n \phi_{\mathrm{B}}+I R_{\mathrm{S}} .
$$

\section{Experimental techniques}

\subsection{Chemical compounds and materials}

ITO coated glass slides (from Sigma-Aldrich) with a surface resistivity of $15-25 \Omega /$ sq were used as the substrates. The chemicals used in cleaning process are acetone $\left(\mathrm{CH}_{3} \mathrm{C}(\mathrm{O}) \mathrm{CH}_{3}, 99.8 \%\right.$, from Merck), ethanol $\left(\mathrm{C}_{2} \mathrm{H}_{6} \mathrm{O}, 99.8 \%\right.$, from Sigma-Aldrich), and 2-propanol $\left(\mathrm{CH}_{3} \mathrm{CH}(\mathrm{OH}) \mathrm{CH}_{3}, 99.8 \%\right.$, from Merck). For thin film and device preparation, high purity materials were used. $N, N^{\prime}$-bis(3-methylphenyl)- $N, N^{\prime}$ diphenylbenzidine (TPD) was used as hole transport layer material (Sigma-Aldrich, Fig. 1). We synthesized the MPPBA [10] and THIBSi [11] molecules according to the previously published procedures. Atomic force microscopy surface characterization was performed in semi-contact (tapping) mode operation using commercial Scanning Probe Microscopy instrument (Solver Pro 7 from MNT-MDT, Russia).

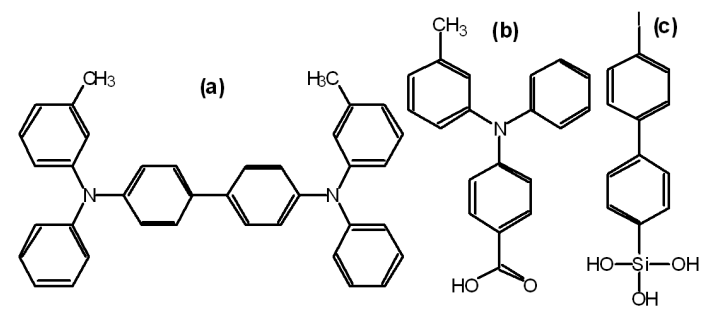

Fig. 1. Chemical structures of TPD (a), MPPBA (b), and THIBSi (c).

\subsection{Schottky device preparations}

The ITO coated glass slides were etched using zinc powder and $20 \% \mathrm{HCl}$ acid solution. Etched ITO substrates were placed in ultrasonic cleaner and purified in highly pure deionized water, acetone, ethanol, 2-propanol and again deionized water, respectively, for $15 \mathrm{~min}$ in each. After drying in $\mathrm{N}_{2}$ stream, oxygen plasma cleaning in a closed system was carried out using $14 \% \quad \mathrm{O}_{2}$ and $86 \%$ Ar gas mixture for $15 \mathrm{~min}$ in order to clean their surfaces from volatile species and organic molecules. Cleaned ITO substrates were immediately dipped into $1 \mathrm{mM}$ ethanol solution of MPPBA and $1 \mathrm{mM}$ chloroform solution of THIBSi. They were kept in this solution at room temperature for $48 \mathrm{~h}$. The substrates were then rinsed with ethanol to remove the residual SAM molecules from the ITO surface and finally they were dried in an organic evaporation system by blowing a stream of $\mathrm{N}_{2}$ gas. The organic HTL material (TPD) was deposited under high vacuum $\left(\approx 10^{-6}\right.$ Torr $)$ with a low deposition rate $(\approx 0.5 \AA / \mathrm{s})$. Finally high purity $\mathrm{Al}$ $(99 \%)$ was thermally evaporated to form the top contact cathode layer with a thickness of $120 \mathrm{~nm}$ at a pressure of $4 \times 10^{-6}$ Torr and a deposition rate of $3 \AA / \mathrm{s}$ in the same evaporation system.

\section{Results and discussions}

\subsection{Atomic force microscopy (AFM)}

We used AFM technique in order to investigate the topography of the bare ITO and SAM modified ITO surfaces in high-resolution mode that demonstrates resolution of fractions in nanometer scale. The measured surface roughness (rms) of the bare ITO surface was found as $3.71 \mathrm{~nm}$. Figure 2 shows surface topography images of (a) bare ITO, (b) MPPBA modified ITO and (c) THIBSi modified ITO surfaces. Island-like structures can be seen after the formation of SAM layer and as a result of coating of MPPBA and THIBSi molecules, surface properties of the ITO surface has changed. The average roughness of MPPBA modified ITO surface was measured as $3.41 \mathrm{~nm}$.

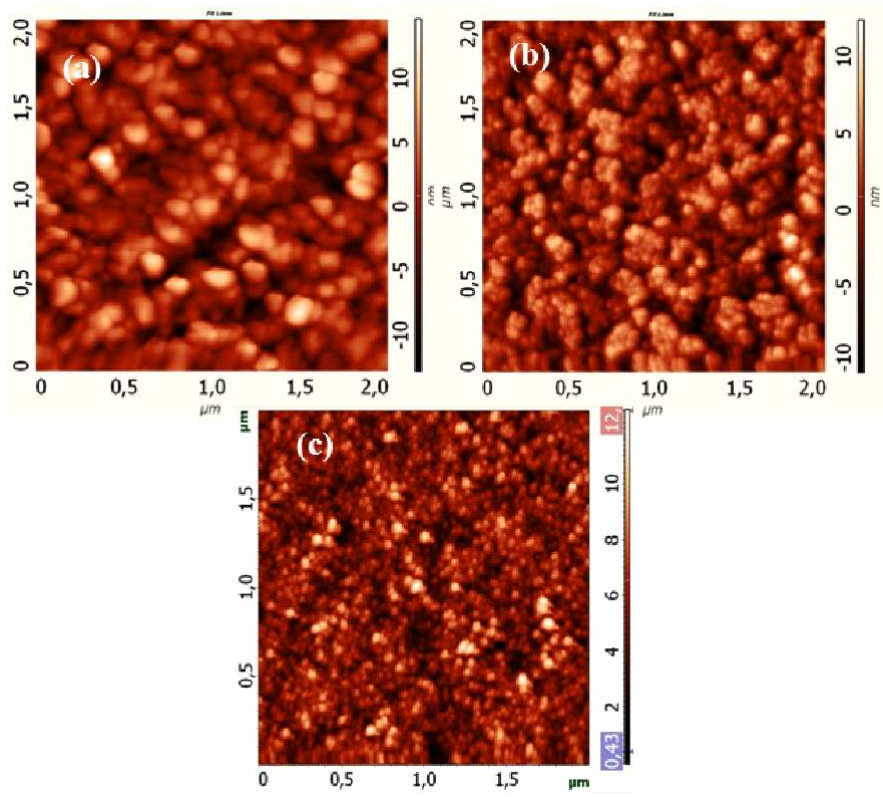

Fig. 2. Surface topography image of bare ITO (a), surface topography image of MPPBA modified ITO (b), and surface topography image of THIBSi modified ITO (c).

\subsection{Schottky thermal injection results}

The Schottky characteristics of the devices were investigated to calculate barrier height after the surface modification with SAM technique and TPD molecules. 
The $I-V$ characteristics of the Schottky devices (ITO/ TPD/Al, ITO/MPPBA/TPD/Al and ITO/THIBSi/ $\mathrm{TPD} / \mathrm{Al}$ ) are shown in Fig. $3 \mathrm{a}-\mathrm{c}$. As the work function and barrier height values of the materials used in the fabrication of the devices are close to each other, plots become symmetric at negative and positive applied voltage intervals (Fig. 3a). The SAM modified ITO devices increase the current with respect to unmodified ITO devices (Fig. 3a).

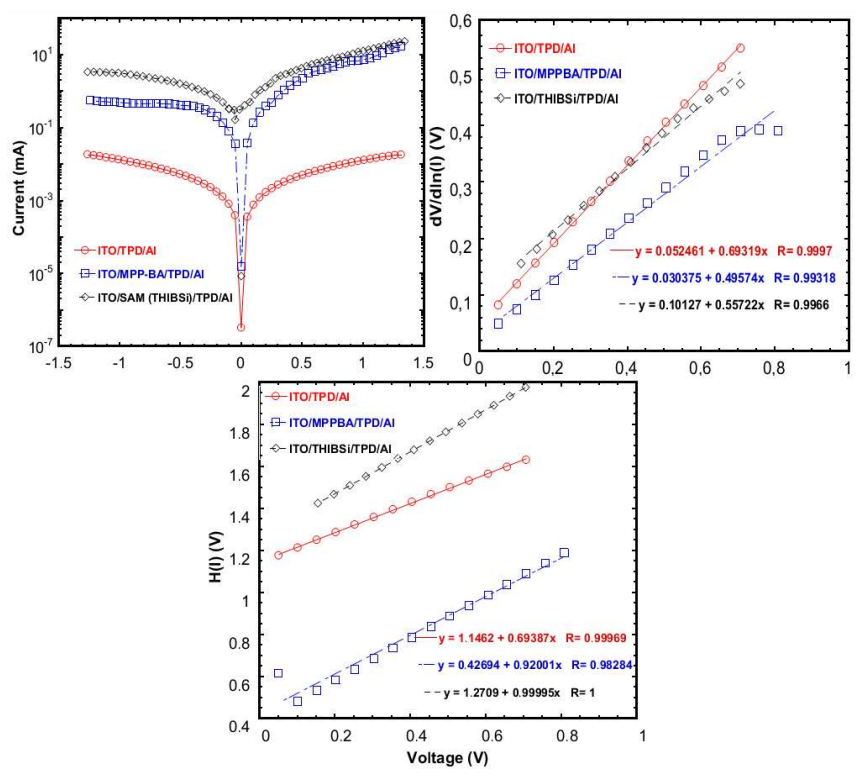

Fig. 3. $I-V$ characteristics of fabricated ITO/ $\mathrm{TPD} / \mathrm{Al}$, ITO/MPPBA/TPD/Al, ITO/THIBSi $\mathrm{TPD} / \mathrm{Al}$ and devices.

The calculated Schottky diode parameters such as ideality factor $n$ and barrier height $\Phi_{\mathrm{B}}$ values are presented in Table. Results show that SAM modification decreases the barrier height and enhances the device performance.

\section{TABLE}

Calculated ideality factor and barrier height values of devices with bare and SAM modified ITO.

\begin{tabular}{l|c|c}
\hline \hline \multicolumn{1}{c|}{ Devices } & $n$ & $\Phi_{\mathrm{B}}[\mathrm{eV}]$ \\
\hline ITO/TPD/Al & 2.03 & 0.56 \\
ITO/MPPBA/TPD/Al & 1.18 & 0.36 \\
ITO/THIBSi/TPD/Al & 3.92 & 0.32
\end{tabular}

\section{Conclusion}

The electrical characteristics of the Schottky devices fabricated from SAM modified and bare ITO glass slides have been investigated by $I-V$ methods. The electrical properties of the modified electrodes showed an enhanced performance as a result of the improvement of charge injection with respect to unmodified electrode device. The ideality factor of diodes is higher than unity. That is because of the occurrence of a non-ideal currentvoltage behavior in the devices higher than unity. The $I-V$ characteristics indicate that electronic parameters of the ITO/MPBA/TPD/Al diode are better than of other devices.

\section{Acknowledgments}

This work was supported by TUBITAK under grant No. TBAG-108T718.

\section{References}

[1] A.J. Heeger, S. Kivelson, J.R. Schrieffer, W.P. Su, Rev. Mod. Phys. 60, 781 (1988) G.J. Ashwell, Molecular Electronics, Research Studies Press, Taunton UK 1992

[2] J. Lei, W. Liang, C.J. Brumlik, C.R. Martin, Synth. Met. 47, 351 (2004)

[3] F. Nüesch, E.W. Forsythe, Q.T. Le, Y. Gao, L.J. Rothberg, J. Appl. Phys. 87, 7973 (2000)

[4] H. Ishii, K. Sugiyama, E. Ito, K. Seki, Adv. Mater. 11, 972 (1999).

[5] R.K. Gupta, R.A. Singh, Compos. Sci. Technol. 65, $677(2005)$ F. Yakuphanoglu, J. Phys. Chem. C 111, $1505(2007)$

[6] J. Cui, Q. Huang, J.G.C. Veinot, H. Yan, T.J. Marks, Adv. Mater. 14, 565 (2002)

[7] J.C. Scott, J. Vac. Sci. Technol. A 21, 521 (2003)

[8] E.H. Rhoderick, R. Williams, Metal-Semiconductor Contacts, Clarendon Press, Oxford 1988.

[9] S. Cheung, N. Cheung, Appl. Phys. Lett. 49, 85 (1986)

[10] M.C. Harris, S.L. Buchwald, J. Org. Chem. 65, 5327 (2000).

[11] M. Ronchi, M. Pizzotti, A. Orbelli Biroli, P. Macchi, E. Lucenti, C. Zucchi, J. Organomet. Chem. 692, 1788 (2007) 